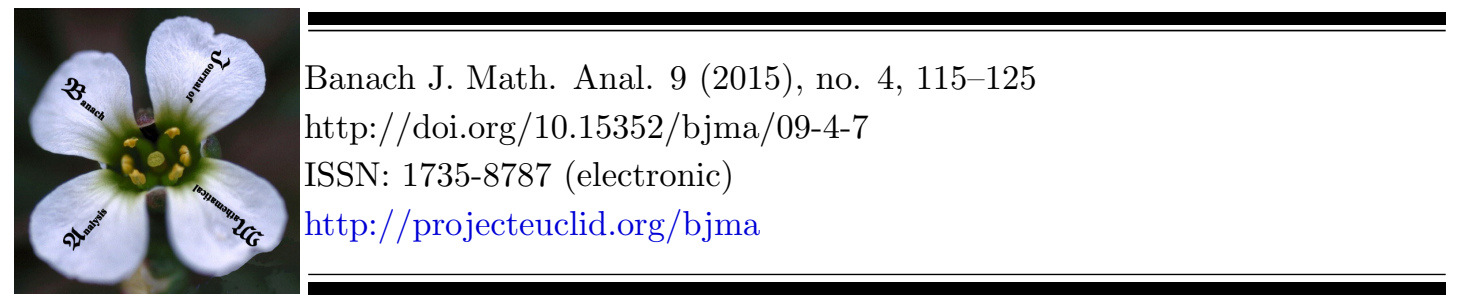

\title{
NONCOMMUTATIVE ORLICZ MODULAR SPACES ASSOCIATED WITH GROWTH FUNCTIONS
}

\author{
ABDUGHENI ABDUREXIT AND TURDEBEK N. BEKJAN* \\ Communicated by Q. Xu
}

\begin{abstract}
We study the noncommutative Orlicz modular spaces associated with growth functions. Some basic properties of such spaces, such as completeness and dominated convergence theorem, are present. Moreover, Young and Clarkson-McCarthy inequalities on these spaces proved.
\end{abstract}

\section{INTRODUCTION}

Let $\mathcal{M}$ be a semi-finite von Neumann algebra equipped with a normal faithful semi-finite trace $\tau$. Given $0<p \leq \infty$ we denote by $L^{p}(\mathcal{M})$ the usual noncommutative $L^{p}$-spaces associated with $(\mathcal{M}, \tau)$. For the theory of noncommutative $L^{p}$-spaces, we refer the readers to $[3,4,10,12]$. We denote the set of all $\tau$-measure operators by $L_{0}(\mathcal{M}, \tau)$, or simply $L_{0}(\mathcal{M})$. We consider the noncommutative Orlicz modular spaces $L^{\Phi}(\mathcal{M})$ associated with a growth functions $\Phi$ (see preliminaries for the definition). About the modular theory we refer the interested readers to $[6,9,11,13]$. It is well-known that Orlicz spaces for convex functions are the generalization of $L_{p}$ spaces for $p \geq 1$. Many authors discuss Orlicz spaces for convex functions( see $[6,9,11,13])$. In this work we study noncommutative Orlicz spaces for the more general case of growth functions. These kinds of noncommutative Orlicz spaces are a generalization of noncommutative $L_{p}$ spaces for $p>0$. In section 1 , we show some results about the growth functions such

Date: Received: Oct. 4, 2014; Accepted: Dec. 29, 2014.

* Corresponding author.

2010 Mathematics Subject Classification. Primary 46L52; Secondary 46L51, 46A80.

Key words and phrases. Clarkson-McCarthy inequalities, growth function, noncommutative Orlicz spaces, modular space, Young inequality. 
as, mainly, characterizations of $\Delta_{2}$ and $\Delta_{\frac{1}{2}}$ conditions, and relationship between general growth functions and concave or convex growth functions.

T. Fack and H. Kosaki in [4] proved the dominated convergence theorems of $\tau$-measurable operators for noncommutative $L^{p}$-spaces. G. Sadeghi proved this theorem for noncommutative Orlicz spaces associated with convex functions in [13]. We extend this result to the noncommutative Orlicz spaces associated with growth functions. This will present in section 2. In section 3, we prove Young and Clarkson-McCarthy inequalities for noncommutative Orlicz spaces associated with growth functions.

\section{RELATIONSHIPS OF GROWTH FUNCTIONS}

Definition 2.1. We say a function $\Phi$ is a growth function, if $\Phi$ is a continuous and nondecreasing function from $[0, \infty)$ onto itself.

In this paper we always assume that for a growth function $\Phi, t \Phi^{\prime}(t)$ is also a growth function. There are many simple examples of growth functions $\Phi$ such that $t \Phi^{\prime}(t)$ are also growth functions. For example $\Phi(t)=t^{p}$, for every $p>0$. There are also some growth functions $\Phi$ such that $t \Phi^{\prime}(t)$ are not growth functions, such as $\Phi(t)=\ln (1+t)$.

For a growth function $\Phi$, set $a=\sup \{t: \Phi(t)=0\}$. Then $a<\infty$ and $\Phi(t)=0$ for all $t \in[0, a]$. Hence we may assume that $\Phi(t)>0$ for all $t>0$ (otherwise replace $\Phi$ by $\Phi(a+\cdot)$ ). For a growth function $\Phi$, we have the following quantitative indices:

$$
p_{\Phi}=\inf _{t>0} \frac{t \Phi^{\prime}(t)}{\Phi(t)}, \quad q_{\Phi}=\sup _{t>0} \frac{t \Phi^{\prime}(t)}{\Phi(t)} .
$$

Definition 2.2. (i) A growth function $\Phi$ obeys $\Delta_{2}$-condition for all $t>0$, often written as $\Phi \in \Delta_{2}$, if there is a constant $K>1$ such that $\Phi(2 t) \leq K \Phi(t)$.

(ii) A growth function $\Phi$ said to satisfy the $\Delta_{\frac{1}{2}}$-condition for all $t>0$, denoted symbolically as $\Phi \in \Delta_{\frac{1}{2}}$, if there is a constant $0<K<1$ such that $\Phi\left(\frac{t}{2}\right) \leq$ $K \Phi(t)$.

By Proposition 1.4 in [1], we have that

$$
\Phi \in \Delta_{2} \Leftrightarrow q_{\Phi}<\infty, \quad \Phi \in \Delta_{\frac{1}{2}} \Leftrightarrow p_{\Phi}>0 .
$$

If $\Phi$ satisfies the $\Delta_{2}$ and $\Delta_{\frac{1}{2}}$ conditions for all $t>0$, we denote symbolically as $\Phi \in \Delta_{2} \cap \Delta_{\frac{1}{2}}$.

Definition 2.3. Growth functions $\Phi_{1}, \Phi_{2}$ are said to be equivalent, denoted by $\Phi_{1} \sim \Phi_{1}$, if there exist positive constants $C_{1}, C_{2}, C_{3}, C_{4}$ such that

$$
C_{1} \Phi_{1}\left(C_{2} t\right) \leq \Phi_{2}(t) \leq C_{3} \Phi_{1}\left(C_{4} t\right), \quad \forall t>0 .
$$

In [1], we proved that if $\Phi_{1} \sim \Phi_{1}$, then $\Phi_{1} \in \Delta_{2} \Leftrightarrow \Phi_{2} \in \Delta_{2}$ and $\Phi_{1} \in \Delta_{\frac{1}{2}} \Leftrightarrow$ $\Phi_{2} \in \Delta_{\frac{1}{2}}$ (see Proposition 1.4).

Proposition 2.4. Let $\Phi$ be a growth function. Then the followings hold. 
(i) For every $p \in\left[0, p_{\Phi}\right], t^{-p} \Phi(t)$ is non-decreasing function and for every $q \in$ $\left[q_{\Phi}, \infty\right), t^{-q} \Phi(t)$ is non-increasing function.

(ii) $\Phi \in \Delta_{2}$ is equivalent to that for a constant $C>1$ there is a constant $K>1$ such that $\Phi(C t) \leq K \Phi(t)$ for all $t>0$.

(iii) $\Phi \in \Delta_{\frac{1}{2}}$ is equivalent to that for a constant $0<C<1$ there is a constant $0<K<1$ such that $\Phi(C t) \leq K \Phi(t)$ for all $t>0$.

Proof. (i) For every $p \in\left(0, p_{\Phi}\right], t^{-p} \Phi(t)$ is non-decreasing function. Indeed,

$$
\left(t^{-p} \Phi(t)\right)^{\prime}=t^{-p-1}\left(t \Phi^{\prime}(t)-p \Phi(t)\right) \geq t^{-p-1}\left(p_{\Phi} \Phi(t)-p \Phi(t)\right) \geq 0 .
$$

Similarly, we get $t^{-q} \Phi(t)$ is non-increasing for every $q \in\left[q_{\Phi}, \infty\right)$.

(ii) Let $\Phi \in \Delta_{2}$. If $C>1$, then by (2.1) and (i),

$$
\Phi(C t) \leq C^{q_{\Phi}} \Phi(t), \quad \forall t>0,
$$

i.e., the desired result holds. Conversely, if there are constants $C>1$ and $K>1$ such that $\Phi(C t) \leq K \Phi(t)$ for all $t>0$, then there is an integer $n \geq 0$ such that $C^{n}<2 \leq C^{n+1}$. It follows that $\Phi(2 t) \leq \Phi\left(C^{n+1} t\right) \leq K^{n+1} \Phi(t)$ and $K^{n+1}>1$, i.e., $\Phi \in \Delta_{2}$.

(iii) Let $\Phi \in \Delta_{\frac{1}{2}}$. If $0<C<1$, then by (2.1) and (i), we get

$$
\Phi(C t) \leq C^{p_{\Phi}} \Phi(t), \quad \forall t>0 .
$$

This implies the desired result. Conversely, let $0<C<1$ and $0<K<1$ such that $\Phi(C t) \leq K \Phi(t)$ for all $t>0$. Then

$$
\frac{1-C}{C} t \Phi^{\prime}(t) \geq \int_{c t}^{t} \frac{s \Phi^{\prime}(s)}{s} d s=\Phi(t)-\Phi(c t) \geq(1-K) \Phi(t) .
$$

So $p_{\Phi} \geq \frac{C}{1-C}(1-K)>0$. By $(2.1), \Phi \in \Delta_{\frac{1}{2}}$.

We let $\Phi^{(\alpha)}(t)=\Phi\left(t^{\alpha}\right)$, where $\alpha$ is a positive real number. Then it is easy to see that $p_{\Phi^{(\alpha)}}=\alpha p_{\Phi}, q_{\Phi(\alpha)}=\alpha q_{\Phi}$.

Theorem 2.5. Let $\Phi$ be a growth function and $\Phi \in \Delta_{\frac{1}{2}} \cap \Delta_{2}$. Set $\Phi_{0}(t)=t \Phi^{\prime}(t)$.

(i) If $q_{\Phi_{0}} \alpha \leq 1$, then $\Phi^{(\alpha)}$ is a concave growth function.

(ii) If $p_{\Phi_{0}} \alpha \geq 1$, then $\Phi^{(\alpha)}$ is a convex growth function.

Proof. (i) Set $a=\sup \left\{t: \Phi^{\prime}(t)=0\right\}$. Then $a<\infty$ and $\Phi(t)=0$ for all $t \in(0, a]$. Hence we may assume that $\Phi^{\prime}(t)>$ for all $t>0$ (otherwise replace $\Phi$ by $\Phi(a+\cdot)$ ).

Since $\Phi_{0}(t)$ is nondecreasing function on $[0, \infty), \Phi^{\prime \prime}(t)$ exists for all $t>0$ except a countable set of points in which we take $\Phi^{\prime \prime}(t)$ as the derivative from the right. Computing we get that

$$
\left(\Phi^{(\alpha)}(t)\right)^{\prime}=\alpha t^{\alpha-1} \Phi^{\prime}\left(t^{\alpha}\right)
$$

and

$$
\begin{aligned}
\left(\Phi^{(\alpha)}(t)\right)^{\prime \prime} & =\alpha(\alpha-1) t^{\alpha-2} \Phi^{\prime}\left(t^{\alpha}\right)+\alpha^{2} t^{2(\alpha-1)} \Phi^{\prime \prime}\left(t^{\alpha}\right) \\
& =\alpha^{2} t^{\alpha-2}\left(t^{\alpha} \Phi^{\prime \prime}\left(t^{\alpha}\right)+\left(1-\frac{1}{\alpha}\right) \Phi^{\prime}\left(t^{\alpha}\right)\right) \\
& =\alpha^{2} t^{\alpha-2} \Phi^{\prime}\left(t^{\alpha}\right)\left(\frac{t^{\alpha} \Phi^{\prime \prime}\left(t^{\alpha}\right)}{\Phi^{\prime}\left(t^{\alpha}\right)}+1-\frac{1}{\alpha}\right) .
\end{aligned}
$$


Since $p_{\Phi} \Phi(t) \leq t \Phi^{\prime}(t) \leq q_{\Phi} \Phi(t)$, we get $\Phi \sim \Phi_{0}$. By Proposition 1.4 of [1], $0<p_{\Phi_{0}} \leq q_{\Phi_{0}}<\infty$. Hence

$$
p_{\Phi_{0}} \leq \frac{t\left(\Phi_{0}(t)\right)^{\prime}}{\Phi_{0}(t)}=\frac{t\left(t \Phi^{\prime}(t)\right)^{\prime}}{t \Phi^{\prime}(t)} \leq q_{\Phi_{0}}, \quad \forall t>0,
$$

so

$$
p_{\Phi_{0}}-1 \leq \frac{t \Phi^{\prime \prime}(t)}{\Phi^{\prime}(t)} \leq q_{\Phi_{0}}-1, \quad \forall t>0 .
$$

It follows that

$$
p_{\Phi_{0}}-1 \leq \frac{t^{\alpha} \Phi^{\prime \prime}\left(t^{\alpha}\right)}{\Phi^{\prime}\left(t^{\alpha}\right)} \leq q_{\Phi_{0}}-1, \quad \forall t>0 .
$$

Since $q_{\Phi_{0}} \alpha \leq 1$, by $(2.2)$ we have that

$$
\left(\Phi^{(\alpha)}(t)\right)^{\prime \prime} \leq \alpha^{2} t^{\alpha} \Phi^{\prime}\left(t^{\alpha}\right)\left(q_{\Phi}-\frac{1}{\alpha}\right) \leq 0, \quad \forall t>0,
$$

which implies that $\Phi^{(\alpha)}$ is a concave growth function.

(ii) It is proved in [1].

\section{NONCOMMUTATIVE ORLICZ SPACES}

Let $\mathcal{M}$ be a semi-finite von Neumann algebra equipped with a normal faithful semi-finite trace $\tau$. Let $L_{0}(\mathcal{M})$ denote the topological $*$-algebra of measurable operators with respect to $(\mathcal{M}, \tau)$. The topology of $L_{0}(\mathcal{M})$ is determined by the convergence in measure.

Definition 3.1. Let $\Phi$ be a growth function. We define the corresponding noncommutative Orlicz space on $(\mathcal{M}, \tau)$ by

$$
L^{\Phi}(\mathcal{M})=\left\{x \in L_{0}(\mathcal{M}): \tau(\Phi(|\lambda x|))<\infty \text { for some } \lambda>0\right\} .
$$

Let a growth function $\Phi$ satisfies the $\Delta_{2}$-condition. By Proposition 2.4, we know that $\tau(\Phi(|\lambda x|))<\infty$ for some $\lambda>0$ are equivalent to $\tau(\Phi(|x|))<\infty$. So $L^{\Phi}(\mathcal{M})=\left\{x \in L_{0}(\mathcal{M}): \tau(\Phi(|x|))<\infty\right\}$ for $\Phi \in \Delta_{2}$.

Definition 3.2. Let $X$ be an arbitrary vector space. A functional $\rho: X \rightarrow[0, \infty]$ is called a modular, if for arbitrary $x, y \in X$,

(i) $\rho(x)=0$ if and only if $x=0$,

(ii) $\rho(\alpha x)=\rho(x)$ for every scalar $\alpha$ with $|\alpha|=1$,

(iii) $\rho(\alpha x+\beta y) \leq \rho(x)+\rho(y)$ for $\alpha, \beta \geq 0$ such that $\alpha+\beta=1$.

We now define a functional $\rho_{\Phi}$ on $L_{0}(\mathcal{M})$ by

$$
\rho_{\Phi}(x)=\tau(\Phi(|x|)), \quad \forall x \in L_{0}(\mathcal{M}) .
$$

It follows from corollary 2.8 of [4] that $\rho_{\Phi}(x)=\tau(\Phi(|x|))=\int_{0}^{\infty} \Phi\left(\mu_{t}(x)\right) d t$.

Theorem 3.3. Let $\Phi$ be a growth function and $\Phi \in \Delta_{\frac{1}{2}} \cap \Delta_{2}$. Then $\rho_{\Phi}$ is a modular on $L_{0}(\mathcal{M})$. 
Proof. It is clear that $\rho_{\Phi}$ satisfies the (i) and (ii) of Definition 3.2. Let $x, y$ be $\tau$-measurable operators, $\alpha, \beta \geq 0$ such that $\alpha+\beta=1$. By Lemma 4.3 of [4] (also see [7]), there exist partial isometries $u, v \in \mathcal{M}$ such that

$$
|\alpha x+\beta y| \leq \alpha u|x| u^{*}+\beta v|y| v^{*} .
$$

Using Theorem 2.5 we find a natural number $n$ such that $\Phi^{\left(\frac{1}{n}\right)}$ is concave growth function. By Proposition 4.6 of [4],

$$
\begin{aligned}
\rho_{\Phi}(\alpha x+\beta y) & =\tau(\Phi(|\alpha x+\beta y|))=\int_{0}^{\infty} \Phi\left(\mu_{t}(\alpha x+\beta y)\right) d t \\
& \leq \int_{0}^{\infty} \Phi\left(\mu_{t}\left(\alpha u|x| u^{*}+\beta v|y| v^{*}\right)\right) d t \\
& =\int_{0}^{\infty} \Phi^{\left(\frac{1}{n}\right)}\left(\left(\mu_{t}\left(\alpha u|x| u^{*}+\beta v|y| v^{*}\right)\right)^{n}\right) d t \\
& =\int_{0}^{\infty} \Phi^{\left(\frac{1}{n}\right)}\left(\mu_{t}\left(\left(\alpha u|x| u^{*}+\beta v|y| v^{*}\right)^{n}\right)\right) d t \\
& \leq \int_{0}^{\infty} \Phi^{\left(\frac{1}{n}\right)}\left(\mu_{t}\left(\alpha\left(u|x| u^{*}\right)^{n}+\beta\left(v|y| v^{*}\right)^{n}\right)\right) d t \\
& \leq \int_{0}^{\infty} \Phi^{\left(\frac{1}{n}\right)}\left(\mu_{t}\left(\alpha\left(u|x| u^{*}\right)^{n}\right)\right) d t+\int_{0}^{\infty} \Phi^{\left(\frac{1}{n}\right)}\left(\mu_{t}\left(\beta\left(v|y| v^{*}\right)^{n}\right)\right) d t \\
& \leq \int_{0}^{\infty} \Phi^{\left(\frac{1}{n}\right)}\left(\alpha \mu_{t}\left(|x|^{n}\right)\right) d t+\int_{0}^{\infty} \Phi^{\left(\frac{1}{n}\right)}\left(\beta \mu_{t}\left(|y|^{n}\right)\right) d t \\
& =\int_{0}^{\infty} \Phi\left(\alpha^{\frac{1}{n}} \mu_{t}(x)\right) d t+\int_{0}^{\infty} \Phi\left(\beta^{\frac{1}{n}} \mu_{t}(y)\right) d t \\
& \leq \int_{0}^{\infty} \Phi\left(\mu_{t}(x)\right) d t+\int_{0}^{\infty} \Phi\left(\mu_{t}(y)\right) d t \\
& =\tau(\Phi(|x|))+\tau(\Phi(|y|)) \\
& =\rho_{\Phi}(x)+\rho_{\Phi}(y) .
\end{aligned}
$$

Thus $\rho_{\Phi}$ is a modular on $L_{0}(\mathcal{M})$.

Theorem 3.4. Let $\Phi$ be a growth function and $\Phi \in \Delta_{\frac{1}{2}} \cap \Delta_{2}$. Then noncommutative Orlicz space $L^{\Phi}(\mathcal{M})$ is $\rho_{\Phi}$-complete.

Proof. Let $\left\{x_{n}\right\}$ be a $\rho_{\Phi}$-Cauchy sequence in $L^{\Phi}(\mathcal{M})$. Then

$$
\rho_{\Phi}\left(x_{n}-x_{m}\right) \rightarrow 0(n, m \rightarrow \infty)
$$

From this we get that for all $t>0$,

$$
\mu_{t}\left(x_{n}-x_{m}\right) \rightarrow 0(n, m \rightarrow \infty) .
$$

Indeed, if $\mu_{t}\left(x_{n}-x_{m}\right) \nrightarrow 0(n, m \rightarrow \infty)$, then there are $\varepsilon_{0}>0, t_{0}>0$ and for any $k \in \mathbb{N}$ there exit $n_{k}, m_{k}>k$ such that

$$
\mu_{t_{0}}\left(x_{n_{k}}-x_{m_{k}}\right) \geq \varepsilon_{0} .
$$


By the right continuity of $\mu_{t}$, there exists $\delta>0$ such that

$$
\mu_{t}\left(x_{n_{k}}-x_{m+k}\right) \geq \frac{\varepsilon_{0}}{2}, \quad \forall t \in\left(t_{0}, t_{0}+\delta\right) .
$$

By the definition of a growth function $\Phi$, we have

$$
\begin{aligned}
\rho_{\Phi}\left(x_{n_{k}}-x_{m_{k}}\right) & =\tau\left(\Phi\left(x_{n_{k}}-x_{m_{k}}\right)\right) \\
& =\int_{0}^{\infty} \Phi\left(\mu_{t}\left(x_{n_{k}}-x_{m_{k}}\right)\right) d t \\
& \geq \int_{t_{0}}^{t_{0}+\delta} \Phi\left(\mu_{t}\left(x_{n_{k}}-x_{m_{k}}\right)\right) d t \\
& \geq \Phi\left(\frac{\varepsilon_{0}}{2}\right) \delta>0 .
\end{aligned}
$$

That is contraction. Thus (3.1) holds.

By Lemma 3.1 of [4], $\left\{x_{n}\right\}$ is a cauchy sequence in $L_{0}(\mathcal{M})$. Since $L_{0}(\mathcal{M})$ is a complete, there exist a $\tau$-measurable operator $x \in L_{0}(\mathcal{M})$ such that $\left\{x_{n}\right\}$ convergence to $x$ in measure topology. By the Lemma 3.4 of [4] and continuity of $\Phi$, we obtain

$$
\begin{aligned}
\rho_{\Phi}(x) & =\tau(\Phi(x))=\int_{0}^{\infty} \Phi\left(\mu_{t}(x)\right) d t \\
& \leq \int_{0}^{\infty} \lim _{n \rightarrow \infty} \inf \Phi\left(\mu_{t}\left(x_{n}\right)\right) d t \\
& \leq \lim _{n \rightarrow \infty} \inf \int_{0}^{\infty} \Phi\left(\mu_{t}\left(x_{n}\right)\right) d t \\
& =\lim _{n \rightarrow \infty} \inf \rho_{\Phi}\left(x_{n}\right) .
\end{aligned}
$$

Similarly,

$$
\rho_{\Phi}\left(x-x_{n}\right) \leq \lim _{m \rightarrow \infty} \inf \rho_{\Phi}\left(x_{m}-x_{n}\right), \quad \forall n \in \mathbb{N} .
$$

On the other hand, since $\Phi \in \Delta_{2}$,

$$
\begin{aligned}
\rho_{\Phi}(x)=\rho_{\Phi}\left(x-x_{n}+x_{n}\right) & \left.\leq \rho_{\Phi}\left(2\left(x-x_{n}\right)\right)+\rho_{\Phi}\left(2 x_{n}\right)\right) \\
& \leq K\left(\rho_{\Phi}\left(x-x_{n}\right)+\rho_{\Phi}\left(x_{n}\right)\right) .
\end{aligned}
$$

This implies $\rho_{\Phi}(x)<\infty$, i.e. $x \in L^{\Phi}(\mathcal{M})$. By (3.2), it follows that the sequence $\left\{x_{n}\right\}$ is $\rho_{\Phi}$-convergent to $x$.

Next, we prove the dominated convergence theorems for $\tau$-measurable operators with respect to the modular $\rho_{\Phi}$.

Theorem 3.5. Let $\Phi$ be a growth function and $\Phi \in \Delta_{\frac{1}{2}} \cap \Delta_{2}$. Let $\left\{x_{n}\right\}$ be a sequence of $\tau$-measurable operators converging to $x$ in the measure topology. Assume that there exist $\tau$-measurable operators $y_{n}, n=1,2, \cdots$ and $y$ in $L^{\Phi}(\mathcal{M})$, satisfying the following conditions:

(i) $\left|x_{n}\right| \leq\left|y_{n}\right|$,

(ii) $\rho_{\Phi}(y)=\lim _{n \rightarrow \infty} \rho_{\Phi}\left(y_{n}\right)$,

(iii) the sequence $\left\{y_{n}\right\}$ converges to $y$ in the measure topology. 
Then $x_{n}$ and $x$ are in $L^{\Phi}(\mathcal{M})$ and

$$
\lim _{n \rightarrow \infty} \rho_{\Phi}\left(x_{n}-x\right)=0 .
$$

Proof. We use the method in the proof of Theorem 3.11 of [13]. Since $\Phi \in \Delta_{2}$,

$$
L^{\Phi}(\mathcal{M})=\left\{x \in L_{0}(\mathcal{M}): \tau(\Phi(|x|))<\infty\right\} .
$$

From the condition (i) and Lemma 2.5 of [4] we know that $\mu_{t}\left(x_{n}\right) \leq \mu_{t}\left(y_{n}\right)$. Therefore for the growth function $\Phi$,

$$
\int_{0}^{\infty} \Phi\left(\mu_{t}\left(x_{n}\right)\right) d t \leq \int_{0}^{\infty} \Phi\left(\mu_{t}\left(y_{n}\right)\right) d t .
$$

Whence

$$
\rho_{\Phi}\left(x_{n}\right) \leq \rho_{\Phi}\left(y_{n}\right) .
$$

This implies that $x_{n} \in L^{\Phi}(\mathcal{M})$, since $y_{n} \in L^{\Phi}(\mathcal{M})$. From the proof of Theorem 3.4, we know that

$$
\rho_{\Phi}(x) \leq \lim _{n \rightarrow \infty} \inf \rho_{\Phi}\left(x_{n}\right) \leq \lim _{n \rightarrow \infty} \inf \rho_{\Phi}\left(y_{n}\right) \leq \rho_{\Phi}(y) .
$$

This inequality ensures that $x \in L^{\Phi}(\mathcal{M})$.

Since $\Phi$ is a growth function and satisfies the $\Delta_{2}$-condition, by the Theorem 2.5 , there is a natural number $n_{0}$ such that $\Phi^{\left(\frac{1}{n_{0}}\right)}$ concave growth function. Hence

$$
\begin{aligned}
\Phi(\alpha+\beta) & =\Phi^{\left(\frac{1}{n_{0}}\right)}\left((\alpha+\beta)^{n_{0}}\right) \leq \Phi^{\left(\frac{1}{n_{0}}\right)}\left(2^{n_{0}-1} \alpha^{n_{0}}+2^{n_{0}-1} \beta^{n_{0}}\right) \\
& \leq \Phi^{\left(\frac{1}{n_{0}}\right)}\left(2^{n_{0}-1} \alpha^{n_{0}}\right)+\Phi^{\left(\frac{1}{n_{0}}\right)}\left(2^{n_{0}-1} \beta^{n_{0}}\right) \\
& \leq \Phi(2 \alpha)+\Phi(2 \beta) \\
& \leq K(\Phi(\alpha)+\Phi(\beta)), \quad \forall \alpha, \beta \geq 0
\end{aligned}
$$

Applying this we obtain that

$$
\begin{aligned}
\Phi\left(\mu_{t}\left(x_{n}-x\right)\right) & \leq \Phi\left(\mu_{\frac{t}{2}}\left(x_{n}\right)+\mu_{\frac{t}{2}}(x)\right) \\
& \leq \Phi\left(\mu_{\frac{t}{2}}\left(y_{n}\right)+\mu_{\frac{t}{2}}(x)\right) \\
& \leq K\left(\Phi\left(\mu_{\frac{t}{2}}\left(y_{n}\right)\right)+\Phi\left(\mu_{\frac{t}{2}}(x)\right)\right)
\end{aligned}
$$

On the other hand, it follows from Lemma 3.1 and 3.4 of [4] that

$$
\lim _{n \rightarrow \infty} \mu_{t}\left(x_{n}-x\right)=0 \quad \text { and } \quad \mu_{\frac{t}{2}}(y) \leq \lim _{n \rightarrow \infty} \inf \mu_{\frac{t}{2}}\left(y_{n}\right)
$$

Hence

$$
\begin{aligned}
& \lim _{n \rightarrow \infty} \inf \left\{K\left[\Phi\left(\mu_{\frac{t}{2}}\left(y_{n}\right)\right)+\Phi\left(\mu_{\frac{t}{2}}(x)\right)\right]-\Phi\left(\mu_{t}\left(x_{n}-x\right)\right)\right\} \\
& \geq K\left[\Phi\left(\mu_{\frac{t}{2}}(y)\right)+\Phi\left(\mu_{\frac{t}{2}}(x)\right)\right] .
\end{aligned}
$$


By usual Fatou Lemma, we have that

$$
\begin{aligned}
& \int_{0}^{\infty} K\left[\Phi\left(\mu_{\frac{t}{2}}(y)\right)+\Phi\left(\mu_{\frac{t}{2}}(x)\right)\right] d t \\
& \leq \int_{0}^{\infty} \lim _{n \rightarrow \infty} \inf \left\{K\left[\Phi\left(\mu_{\frac{t}{2}}\left(y_{n}\right)\right)+\Phi\left(\mu_{\frac{t}{2}}(x)\right)\right]-\Phi\left(\mu_{t}\left(x_{n}-x\right)\right)\right\} d t \\
& \leq \lim _{n \rightarrow \infty} \inf \int_{0}^{\infty}\left\{K\left[\Phi\left(\mu_{\frac{t}{2}}\left(y_{n}\right)\right)+\Phi\left(\mu_{\frac{t}{2}}(x)\right)\right]-\Phi\left(\mu_{t}\left(x_{n}-x\right)\right)\right\} d t .
\end{aligned}
$$

From the assumption (ii) and $x, y \in L^{\Phi}(\mathcal{M})$, we get

$$
-\lim _{n \rightarrow \infty} \sup \int_{0}^{\infty} \Phi\left(\mu_{t}\left(x_{n}-x\right)\right) d t \geq 0 .
$$

That is $\lim _{n \rightarrow \infty} \rho_{\Phi}\left(x_{n}-x\right)=0$.

Corollary 3.6. Let $\Phi$ be a growth function and $\Phi \in \Delta_{\frac{1}{2}} \cap \Delta_{2}$. Let $\left\{x_{n}\right\}$ be a sequence of $\tau$-measurable operators converging to $x$ in the measure topology. If there exists an operator $y \in L^{\Phi}(\mathcal{M})$ such that $\left|x_{n}\right| \leq y$ for $n=1,2, \cdots$, then

$$
\lim _{n \rightarrow \infty} \rho_{\Phi}\left(x_{n}\right)=\rho_{\Phi}(x) .
$$

\section{The Clarkson-MCCarthy inequalities}

In this section we extend Young and Clarkson-McCarthy inequalities on noncommutative Orlicz modular space associated with growth functions.

The classical Young inequality for two nonnegative real numbers $a, b$, is

$$
a b \leq \frac{a^{p}}{p}+\frac{b^{q}}{q}
$$

where $p, q>1$ are such that $p^{-1}+q^{-1}=1$. We have the following result.

Theorem 4.1. Let $\Phi$ be a growth function and $\Phi \in \Delta_{\frac{1}{2}} \cap \Delta_{2}$. Let $p, q \geq 1$ such that $p^{-1}+q^{-1}=1$. If $x \in L^{\Phi^{(p)}}(\mathcal{M})$ and $y \in L^{\Phi^{(q)}}(\mathcal{M})$, then

$$
\rho_{\Phi}(x y) \leq \frac{1}{p} \rho_{\Phi^{(p)}}(x)+\frac{1}{q} \rho_{\Phi^{(q)}}(y) .
$$

Consequently, $x y \in L^{\Phi}(\mathcal{M})$.

Proof. By the classical Young inequality,

$$
\mu_{t}(a) \mu_{t}(b) \leq \frac{\mu_{t}(a)^{p}}{p}+\frac{\mu_{t}(b)^{q}}{q}, \quad \forall a, b \in L_{0}(\mathcal{M})
$$

By Theorem 2.5, there is a $n \in \mathbb{N}$ such that $\Phi^{(n)}$ is a convex growth function. Hence $\Phi\left(e^{t}\right)=\Phi^{(n)}\left(e^{\frac{t}{n}}\right)$ is also convex function. Then, by (iii) of Theorem 4.2 in 
[4] and (4.1), we get

$$
\begin{aligned}
\rho_{\Phi}(x y) & =\int_{0}^{\infty} \Phi\left(\mu_{t}(x y)\right) d t \\
& \leq \int_{0}^{\infty} \Phi\left(\mu_{t}(x) \mu_{t}(y)\right) d t \\
& =\int_{0}^{\infty} \Phi^{(n)}\left(\mu_{t}\left(|x|^{\frac{1}{n}}\right) \mu_{t}\left(|y|^{\frac{1}{n}}\right)\right) d t \\
& \leq \int_{0}^{\infty} \Phi^{(n)}\left(\frac{\mu_{t}\left(|x|^{\frac{1}{n}}\right)^{p}}{p}+\frac{\mu_{t}\left(|y|^{\frac{1}{n}}\right)^{q}}{q}\right) d t \\
& \leq \frac{1}{p} \int_{0}^{\infty} \Phi^{(n)}\left(\mu_{t}(x)^{\frac{p}{n}}\right) d t+\frac{1}{q} \int_{0}^{\infty} \Phi^{(n)}\left(\mu_{t}(y)^{\frac{q}{n}}\right) d t \\
& =\frac{1}{p} \tau\left(\Phi^{(p)}(|x|)\right)+\frac{1}{q} \tau\left(\Phi^{(q)}(|y|)\right) \\
& =\frac{1}{p} \rho_{\Phi^{(p)}}(x)+\frac{1}{q} \rho_{\Phi^{(q)}}(y) .
\end{aligned}
$$

This is the desired result.

The following is a generalization of Clarkson-McCarthy inequalities (see [2, 5, 8]).

Theorem 4.2. Let $\Phi$ be a growth function and $\Phi \in \Delta_{\frac{1}{2}} \cap \Delta_{2}$. Set $\Phi_{0}(t)=t \Phi^{\prime}(t)$.

(i) If $p_{\Phi_{0}} \geq 2$, then for $x, y \in L^{\Phi}(\mathcal{M})$, we have

$$
\rho_{\Phi}(x+y)+\rho_{\Phi}(x-y) \geq 2\left(\rho_{\Phi}(x)+\rho_{\Phi}(y)\right) .
$$

Consequently,

$$
\rho_{\Phi}(x+y)+\rho_{\Phi}(x-y) \leq 2^{-1}\left(\rho_{\Phi}(2 x)+\rho_{\Phi}(2 y)\right) .
$$

(ii) If $q_{\Phi_{0}} \leq 2$, then for $x, y \in L^{\Phi}(\mathcal{M})$, we have

$$
\rho_{\Phi}(x+y)+\rho_{\Phi}(x-y) \geq 2^{-1}\left(\rho_{\Phi}(2 x)+\rho_{\Phi}(2 y)\right) .
$$

Consequently,

$$
\rho_{\Phi}(x+y)+\rho_{\Phi}(x-y) \leq 2\left(\rho_{\Phi}(x)+\rho_{\Phi}(y)\right) .
$$

Proof. (i) By the proof of Theorem 2.5, we know that $\Phi^{\left(\frac{1}{2}\right)}$ is a convex growth function. We know that for a convex function,

$$
\Phi^{\left(\frac{1}{2}\right)}(\alpha)+\Phi^{\left(\frac{1}{2}\right)}(\beta) \geq 2 \Phi^{\left(\frac{1}{2}\right)}\left(\frac{\alpha+\beta}{2}\right) .
$$


Using (4.2), (iii) of Theorem 4.4 and (ii) of Proposition 4.6 in [4] we obtain that

$$
\begin{aligned}
\rho_{\Phi}(x+y)+\rho_{\Phi}(x-y) & =\int_{0}^{\infty} \Phi\left(\mu_{t}(x+y)\right) d t+\int_{0}^{\infty} \Phi\left(\mu_{t}(x-y)\right) d t \\
& =\int_{0}^{\infty} \Phi^{\left(\frac{1}{2}\right)}\left(\mu_{t}(x+y)^{2}\right) d t+\int_{0}^{\infty} \Phi^{\left(\frac{1}{2}\right)}\left(\mu_{t}(x-y)^{2}\right) d t \\
& \geq 2 \int_{0}^{\infty} \Phi^{\left(\frac{1}{2}\right)}\left(\frac{\mu_{t}(x+y)^{2}+\mu_{t}(x-y)^{2}}{2}\right) d t \\
& \geq 2 \int_{0}^{\infty} \Phi^{\left(\frac{1}{2}\right)}\left(\mu_{t}\left(\frac{|x+y|^{2}+|x-y|^{2}}{2}\right)\right) d t \\
& =2 \int_{0}^{\infty} \Phi^{\left(\frac{1}{2}\right)}\left(\mu_{t}\left(\frac{(x+y)^{*}(x+y)+(x-y)^{*}(x-y)}{2}\right)\right) d t \\
& =2 \int_{0}^{\infty} \Phi^{\left(\frac{1}{2}\right)}\left(\mu_{t}\left(|x|^{2}+|y|^{2}\right)\right) d t \\
& =2 \tau\left(\Phi^{\left(\frac{1}{2}\right)}\left(|x|^{2}+|y|^{2}\right)\right) \\
& \geq 2\left(\tau\left(\Phi^{\left(\frac{1}{2}\right)}\left(|x|^{2}\right)\right)+2 \tau\left(\Phi^{\left(\frac{1}{2}\right)}\left(|y|^{2}\right)\right)\right) \\
& =2\left(\rho_{\Phi}(x)+\rho_{\Phi}(y)\right) .
\end{aligned}
$$

Replaced $x$ and $y$ by $x+y$ and $x-y$, then we obtain

$$
\rho_{\Phi}(x+y)+\rho_{\Phi}(x-y) \leq 2^{-1}\left(\rho_{\Phi}(2 x)+\rho_{\Phi}(2 y)\right) .
$$

(ii) By Theorem 2.5, we know that $\Phi^{\left(\frac{1}{2}\right)}$ is a concave growth function. Using (i) of Theorem 4.6 in [4] we get

$$
\begin{aligned}
\rho_{\Phi}(x+y) & +\rho_{\Phi}(x-y)=\int_{0}^{\infty} \Phi\left(\mu_{t}(x+y)\right) d t+\int_{0}^{\infty} \Phi\left(\mu_{t}(x-y)\right) d t \\
& =\int_{0}^{\infty} \Phi^{\left(\frac{1}{2}\right)}\left(\mu_{t}(x+y)^{2}\right) d t+\int_{0}^{\infty} \Phi^{\left(\frac{1}{2}\right)}\left(\mu_{t}(x-y)^{2}\right) d t \\
& \geq \int_{0}^{\infty} \Phi^{\left(\frac{1}{2}\right)}\left(\mu_{t}\left(|x+y|^{2}+|x-y|^{2}\right)\right) d t \\
& =\int_{0}^{\infty} \Phi^{\left(\frac{1}{2}\right)}\left(\mu_{t}\left(2|x|^{2}+2|y|^{2}\right)\right) d t \\
& =\int_{0}^{\infty} \Phi^{\left(\frac{1}{2}\right)}\left(\mu_{t}\left(\frac{|2 x|^{2}+|2 y|^{2}}{2}\right)\right) d t \\
& \geq 2^{-1}\left[\tau\left(\Phi^{\left(\frac{1}{2}\right)}\left(|2 x|^{2}\right)\right)+\tau\left(\Phi^{\left(\frac{1}{2}\right)}\left(|2 y|^{2}\right)\right)\right] \\
& =2^{-1}\left(\rho_{\Phi}(2 x)+\rho_{\Phi}(2 y)\right) .
\end{aligned}
$$

Replace $x$ and $y$ by $\frac{x+y}{2}$ and $\frac{x-y}{2}$, we get that

$$
\tau(\Phi(|x+y|))+\tau(\Phi(|x-y|)) \leq 2(\tau(\Phi(|x|))+\tau(\Phi(|y|))) .
$$

Acknowledgement. The authors are grateful to the referee for his/her valuable comments. T.N. Bekjan is partially supported by NSFC grant No.11371304. 


\section{REFERENCES}

1. A. Abdurexit and T.N. Bekjan, Noncommutative Orlicz-Hardy spaces associated with growth functions, J. Math. Anal. Appl. 420 (2014), no. 1, 824-834.

2. R. Bhatia and J.A.R. Holbrook, On the Clarkson-McCarthy inequalities, Math. Ann. 281 (1988), 7-12.

3. T. Fack, Sur la notion de valeur caracteristique, J. Operator Theory 7 (1982), 307-333.

4. T. Fack and H. Kosaki, Generalized s-numbers of $\tau$-measure operators, Pacific J. Math. 123 (1986), 269-300.

5. O. Hirzallah and F. Kittaneh, Non-commutative Clarkson inequalities for unitarily invariant norms, Pacific J. Math. 202 (2002), 363-369.

6. M.A. Khamsi and W.M. Kozlowski, Some geometrical properties and fixed point theorems in Orlicz spaces, J. Math. Anal. Appl. 155(2) (1991), 393-412.

7. H. Kosaki, On the continuity of the map $\varphi \rightarrow|\varphi|$ from the predual of a $W^{*}$-algebra, J. Funct. Anal. 59 (1984), 123-131.

8. C.A. McCarthy, $C_{p}$, Israel J. Math. 5 (1967), 249-271.

9. J. Musielak, Orlicz spaces and modular spaces, in: Lecture Notes in Math. Vol.1034, Springer-Verlag, 1983.

10. E. Nelson, Notes on noncommutative integration, J. Funct. Anal. 15 (1974), 103-116.

11. W. Orlicz, On a class of operators over the space of integeable functions, Studia Math. 14 (1954), 302-309.

12. G. Pisier and Q. Xu, Noncommutative $L^{p}-$ spaces, Handbook of the geometry of Banach spaces 2(2003), 1459-1517.

13. G. Sadeghi, Non-commutative Orlicz spaces associated to a modular on $\tau$-measurable operators, J. Math. Anal. Appl. 395 (2012), 705-715.

College of Mathematics and Systems Sciences, Xinjiang University, Urumqi 830046, CHINA

E-mail address: abdugheni.a@gmail.com

E-mail address: bekjant@yahoo.com 